\title{
Compositional and predicted functional analysis of the gut microbiota of Radix auricularia (Linnaeus) via high-throughput Illumina sequencing
}

\author{
Zongfu Hu ${ }^{1,2,3}{ }^{\text {, Xi Chen }}{ }^{2}$, Jie Chang ${ }^{1}$, Jianhua Yu ${ }^{1}$, Qing Tong ${ }^{2}$, Shuguo Li ${ }^{1}$, Huaxin Niu ${ }^{\text {Corresp. }}{ }^{1}$ \\ ${ }^{1}$ College of Animal Science and Technology, Inner Mongolia University for Nationalities, Tongliao, People's Republic of China \\ 2 College of Animal Science and Technology, Northeast Agricultural University, Harbin, People's Republic of China \\ 3 Inner Mongolia Key Laboratory of Toxicant Monitoring and Toxicology, Tongliao, People's Republic of China \\ Corresponding Author: Huaxin Niu \\ Email address: niuhx@imun.edu.cn
}

Due to its wide distribution across the world, the snail Radix auricularia plays a central role in the transferal of energy and biomass by consuming plant biomass in freshwater systems. The gut microbiota are involved in the nutrition, digestion, immunity, and development of snails, particularly for cellulolytic bacteria, which greatly contribute to the digestion of plant fiber. For the first time, this study characterized the gut bacterial communities of $R$. auricularia, as well as predicted functions, using the Illumina Miseq platform to sequence 16S rRNA amplicons. Both juvenile snails (defined as: JS) and adult snails (defined as: AS) were sampled. The obtained 251,072 sequences were rarefied to 214,584 sequences and clustered into 1,196 operational taxonomic units (OTUs) with $97 \%$ sequence identity. The predominant phyla were Proteobacteria (JS: 36.0\%, AS: 31.6\%) and Cyanobacteria (JS: 16.3\%, AS: 19.5\%), followed by Chloroflexi (JS: 9.7\%, AS: 13.1\%), Firmicutes (JS: 14.4\%, AS: 6.7\%), Actinobacteria (JS: 8.2\%, AS: 12.6\%), and Tenericutes (JS: $7.3 \%$, AS: $6.2 \%$ ). The phylum Cyanobacteria may have originated from the plant diet instead of the gut microbiome. Fifty-two bacterial families and 55 genera were found with $>1 \%$ abundance in at least one sample. A large number of species could not be successfully identified, which could indicate the detection of novel ribotypes or result from insufficient availability of snail microbiome data. The core microbiome consisted of 469 OTUs, representing $88.4 \%$ of all sequences. Furthermore, the predicted function of bacterial community of $R$. auricularia performed by PICRUSt suggests that functions related to metabolism and environmental information processing were enriched. The abundance of carbohydrate suggests a strong capability of the gut microbiome to digest lignin. Our results indicate an abundance of bacteria in both JS and AS, and thus, the bacteria in $R$. auricularia gut form a promising source for novel enzymes, such as, cellulolytic enzymes that may be useful for biofuel production. Furthermore, searching for 
xenobiotic biodegradation bacteria may be a further important application of these snails. 


\section{Composition and predicted Functional analysis of the gut}

\section{2 microbiota of Radix auricularia (Linnaeus) via high-}

\section{3 throughput Illumina sequencing}

5 Zongfu $\mathrm{Hu}^{1,2,3}$, Xi Chen ${ }^{2}$, Jie Chang ${ }^{1}$, Jianhua $\mathrm{Yu}^{1}$, Qing Tong ${ }^{2}$, Shuguo $\mathrm{Li}^{1}$, Huaxin $\mathrm{Niu}^{1 *}$

$6{ }^{1}$ College of Animal Science and Technology, Inner Mongolia University for Nationalities,

7 Tongliao, People's Republic of China

$8{ }^{2}$ College of Animal Science and Technology, Northeast Agricultural University, Haerbin,

9 People's Republic of China

10 3'Inner Mongolia Key Laboratory of Toxicant Monitoring and Toxicology, Tongliao, People's

11 Republic of China

12 Corresponding Author:

13 Huaxin Niu

14 Email address: niuhx@imun.edu.cn 


\section{Compositional and predicted functional analysis of the gut microbiota of}

\section{Radix auricularia (Linnaeus) via high-throughput Illumina sequencing}

23 Zongfu Hu${ }^{1,2,3}$, Xi Chen ${ }^{2}$, Jie Chang ${ }^{1}$, Jianhua Yu ${ }^{1}$, Qing Tong ${ }^{2}$, Shuguo $\mathrm{Li}^{1}$, Huaxin Niu ${ }^{*}$

$24{ }^{1}$ College of Animal Science and Technology, Inner Mongolia University for Nationalities,

25 Tongliao, People's Republic of China

$26{ }^{2}$ College of Animal Science and Technology, Northeast Agricultural University, Haerbin,

27 People's Republic of China

28 3Inner Mongolia Key Laboratory of Toxicant Monitoring and Toxicology, Tongliao, People's

29 Republic of China

\section{ABSTRACT}

32 Due to its wide distribution across the world, the snail Radix auricularia plays a central role in

33 the transferal of energy and biomass by consuming plant biomass in freshwater systems. The gut

34 microbiota are involved in the nutrition, digestion, immunity, and development of snails,

35 particularly for cellulolytic bacteria, which greatly contribute to the digestion of plant fiber. For

36 the first time, this study characterized the gut bacterial communities of $R$. auricularia, as well as

37 predicted functions, using the Illumina Miseq platform to sequence 16S rRNA amplicons. Both

38 juvenile snails (defined as: JS) and adult snails (defined as: AS) were sampled. The obtained

39251,072 sequences were rarefied to 214,584 sequences and clustered into 1,196 operational 40 taxonomic units (OTUs) with $97 \%$ sequence identity. The predominant phyla were

41 Proteobacteria (JS: 36.0\%, AS: 31.6\%) and Cyanobacteria (JS: 16.3\%, AS: 19.5\%), followed by

42 Chloroflexi (JS: 9.7\%, AS: 13.1\%), Firmicutes (JS: 14.4\%, AS: 6.7\%), Actinobacteria (JS: 8.2\%,

43 AS: 12.6\%), and Tenericutes (JS: 7.3\%, AS: 6.2\%). The phylum Cyanobacteria may have 
44 originated from the plant diet instead of the gut microbiome. Fifty-two bacterial families and 55

45 genera were found with $>1 \%$ abundance in at least one sample. A large number of species could

46 not be successfully identified, which could indicate the detection of novel ribotypes or result

47 from insufficient availability of snail microbiome data. The core microbiome consisted of 469

48 OTUs, representing $88.4 \%$ of all sequences. Furthermore, the predicted function of bacterial

49 community of $R$. auricularia performed by PICRUSt suggests that functions related to

50 metabolism and environmental information processing were enriched. The abundance of

51 carbohydrate suggests a strong capability of the gut microbiome to digest lignin. Our results

52 indicate an abundance of bacteria in both JS and AS, and thus, the bacteria in $R$. auricularia gut

53 form a promising source for novel enzymes, such as, cellulolytic enzymes that may be useful for

54 biofuel production. Furthermore, searching for xenobiotic biodegradation bacteria may be a 55 further important application of these snails.

\section{INTRODUCTION}

58 Radix auricularia (Linnaeus, 1758), a pulmonate snail, is naturally distributed in freshwater systems across both Europe and Asia (Stift et al., 2004; Vasileva, 2012). As a primary consumer, snails are common in freshwater systems, and both their energy and biomass can be transferred to fish, turtles, water birds, and mammals (Dewitt et al., 1999; Eckblad, 2010). In addition to

62 their role in the ecosystem, $R$. auricularia are intermediate hosts for many parasites (e.g. flukes), which are harmful to cattle, birds, and humans (Soldánová et al., 2010; Bargues et al., 2001).

The gut bacteria of snails or other animals are involved in multiple physiological processes

65 of their hosts, primarily including digestion, nutrition, development, reproduction, immunity, and 66 environmental resistance (Nicolai et al., 2015; Aronson et al., 2016; Sommer \& Bäckhed, 2013; 
67 Pinheiro et al., 2015; Nayak, 2010). The capacity of decomposing lignocellulosic or pectic 68 biomass increases their ability to utilize a variety of plant biomass, such as algae, water weeds, 69 and leaf litters (Schamp et al., 2010; Vasileva, 2012). Beyond their own digestive enzymes, 70 snails also utilize vast amounts of additional enzymes, secreted by bacterial activity within their 71 gut, which assists in the digestion of up to $60-80 \%$ of the consumed plant fiber (Charrier et al., 72 2006). Cellulase secreted by snail gut bacteria is also used for industrial processes, including the 73 production of biofuels from plant feedstocks (Cardoso et al., 2012a; Pinheiro et al., 2015; Pawar et al., 2015). Their use is more economic and eco-friendly than the use of acid hydrolysis 75 and thermochemical methods (Hamelinck et al., 2005). However, the currently available knowledge of the bacterial communities of the snail gut is limited.

Many factors can influence the composition of the bacterial community of snail and other animals, and previous studies have shown that prominent factors include the diet, season, pathogens, and physiological diseases (Cardoso et al., 2012b; Nicolai et al., 2015; Pawar et al., 2012; Stephens et al., 2016; Chandler et al., 2014). Furthermore, as reported for other animals (zebra fish, bovine, Atlantic salmon), gut microbiomes are also influenced by host development and growth stage (Jami et al., 2013; Nistal et al., 2012; Stephens et al., 2016; Llewellyn et al., 2015). A typical example is the Atlantic salmon (Salmo salar): its gut bacterial communities are more strongly influenced by life-cycle stage than by geography (Llewellyn et al., 2015). composition between sexual and asexual snails, suggesting that reproductive mode influences microbiome composition, which way this relationship goes is still unclear (Vesbach et al., 2016). 
90 reproduction, chiefly in improving spermatogenesis, oocyte maturation, and fecundity

91 (Gioacchini et al., 2011; Carnevali et al., 2017). Therefore, one aim of this study is to

92 understand if the development or growth affects gut microbial communities as well as their 93 functions.

94 In summary, gut bacteria in snails are relevant for physiological processes, the ecosystem, 95 and industrial processes. Consequently, their diversity and function are worth exploring. 96 Although few studies focused on the gut bacterial community of terrestrial snails (Cardoso et al., 97 2012a; Pawar et al., 2012; Nicolai et al., 2015; Charrier et al., 2006), studies of freshwater 98 snails are rare. Here, we characterized the whole profile of the gut bacterial community of $R$. 99 auricularia at different growth stages (juvenile and adult stage) and explored the roles of the 100 obtained gut bacteria in other systems of the snail or the environment via functional prediction of 101 bacteria metagenomic data. The results of this study fill an important gap in our knowledge of 102 mollusks and provide important hints about their potential for technological applications and 103 ecologic significance.

\section{MATERIALS AND METHODS}

106 Research permits were provided by the Forestry Bureau of Tongliao (TL218) and by the Inner

107 Mongolia University for Nationalities' Institutional Animal Use and Care Committee (2016108 IMUN-029).

\section{Sample collection}

110 R. auricularia snails were collected on July 23, 2017 from a pond in Tongliao, Inner Mongolia, 111 China $\left(43^{\circ} 38^{\prime} 2.184^{\prime \prime} \mathrm{N} ; 122^{\circ} 15^{\prime} 43.9632^{\prime \prime} \mathrm{E}\right)$. The depth of the pond was approximately $0.5 \mathrm{~m}$.

112 After transport to the laboratory, all snails were measured and selected for grouping based on 
113 developmental stage. Snails with $9.7 \pm 0.5 \mathrm{~mm}$ shell height were preliminarily classified as

114 adults and pooled into the adult snail group (AS). Snails with $5.5 \pm 0.4 \mathrm{~mm}$ shell height were

115 preliminarily classified as juvenile snails and pooled into the juvenile snail group (JS). Then, the

116 snails were further selected by gonad development: juvenile snails with small and thin gonad;

117 adult snails with full gonads and intumescent, transparent egg mass (Vasileva, 2012; Dikkeboom

118 et al., 1985; Vesbach et al., 2016).

119 Snails were first washed with tap water and then washed with sterile water. $70 \%$ ethanol

120 was used to wipe the snail shells. Then, the snails were anaesthetized with MS-222 (Sigma, St.

121 Louis, MO, USA) and all dissections were performed aseptically, using sterile instruments. Part

122 of the marginal shells were carefully broken, and removed to expose the soft body, and then, the

123 whole soft body was removed from shells and washed by sterile water. The digestive tract was

124 carefully isolated from the body, and then, the portion of gut was collected from the stomach

125 (excluding the stomach) to the anus of the digestive tract (Fig. S1). Meanwhile the snails were

126 further selected according to their gonad development. After sampling and classifying, we ended

127 up with four snails from each group. The total time required for collection and dissection did not

128 exceed two hours. The gut and its contents were carefully collected into plastic cryo-tubes, flash

129 frozen in liquid nitrogen, and stored at $-80^{\circ} \mathrm{C}$ until further analysis.

\section{DNA extraction and PCR amplification}

131 We extracted the genomic DNA using the FastDNA ${ }^{\circledR}$ Spin Kit for Soil (MP Biomedical, USA)

132 according to the manufacturer's instructions. DNA yield and quality are shown in Table 1. After

133 size measure, dissection, we have six samples for each of the AS/JS groupings. Two of each

134 stage were filtered after DNA extraction due to low yield, so we finally get four samples of each

135 stage for sequencing. The $338 \mathrm{~F} / 806 \mathrm{R}$ primer set, targeting the $\mathrm{V} 3-4$ region of the bacterial $16 \mathrm{~S}$ 
136 rRNA gene, was used for PCR amplification as described in Dennis et al (2013). 12-bp barcodes

137 were designed on primers to recognize the sequences of different samples. PCR amplification

138 was performed using the TransStart ${ }^{\circledR}$ FastPfu system (Transgen biotech) (Ma et al., 2014). The

139 following PCR cycle conditions were used: 1 cycle of $95^{\circ} \mathrm{C}$ for 3 min, 27 cycles of $95^{\circ} \mathrm{C}$ for 30

$140 \mathrm{~s}, 55^{\circ} \mathrm{C}$ for $30 \mathrm{~s}$, and $72{ }^{\circ} \mathrm{C}$ for $45 \mathrm{~s}$, and a final extension at $72{ }^{\circ} \mathrm{C}$ for $10 \mathrm{~min}$. Each $20 \mu \mathrm{L}$

141 reaction mixture consisted of $4.0 \mu \mathrm{L}$ of $5 \times$ FastPfu Buffer, $2 \mu \mathrm{L}$ of deoxynucleoside triphosphate

142 (dNTPs) mix (2.5 mM each), $0.4 \mu \mathrm{L}$ of FastPfu DNA Polymerase, $10 \mathrm{ng}$ of template DNA, 0.8

$143 \mu \mathrm{L}$ of Forward Primer 338F $(5 \mu \mathrm{M}), 0.8 \mu \mathrm{L}$ of Forward Primer $806 \mathrm{R}(5 \mu \mathrm{M}), 0.2 \mu \mathrm{L}$ of BSA,

144 then, the remaining volume was filled to $20 \mu 1$ using double-distilled water.

\section{Illumina amplicon sequencing}

146 PCR products were purified using a Trans PCR Purifcation kit and quantified using the

147 QuantiFluor ${ }^{\text {TM}}-$ ST System. Each sample was mixed in equimolar amounts. Then sequence

148 libraries were prepared using the NEB Next巴 ${ }^{\circledR}$ Ultra $^{\mathrm{TM}}$ DNA Library Prep Kit for Illumina (New

149 England Biolabs Inc., Ipswich, MA, USA) according to the manufacturer's instructions. The

150 library quality was assessed by spectrophotometry and 300 bp paired-end sequences were

151 generated on an Illumina Miseq platform PE300 (Illumina Corporation, San Diego, USA) with

152 the 600-cycle MiSeq Reagent Kit v3 (Illumina, San Diego, CA, USA) at the Shangai Majorbio

153 Bio-Pharm Technology co., Ltd. (Shanghai, China).

154 Sequence data of all samples were deposited in the NCBI Sequence Read Archive (SRA)

155 under the BioProject number PRJNA438016.

\section{Data analysis}

157 The paired-end sequences were merged into a single sequence with a length of $434 \mathrm{bp}$ using 158 FLASH (Magoc \& Salzberg, 2011). The QIIME (version 1.17) pipeline was used to eliminate 
159 low quality sequences (i.e. those with $>6 \mathrm{bp}$ of homopolymers, primer mismatches, or mean

160 quality score lower than 25) (Caporaso et al., 2010). Chimeric sequences were removed via

161 HCHIME (Edgar et al., 2011). Then, operational taxonomic units (OTUs) were clustered using

162 Usearch (version $7.1 \mathrm{http}: / /$ drive5.com/uparse/) (Edgar et al., 2011) at a 97\% identity threshold.

163 The number of sequences per sample ranged from 27650 to 37290 . We rarefied each sample to

16426,823 sequences, and 100 iterations of the Usearch rarefaction did not quantitatively change

165 results. We used one of the rarefactions at random among 100 iterations to generate OTUs to

166 represent a table that included the resulting 214,584 sequences to be used in all subsequent

167 analyses. Alpha diversity was analyzed via indices of community diversity (Shannon and

168 Simpson) and community richness (Ace, Chao, and Sobs) using mothur software

169 (http://www.mothur.org/) (Schloss et al., 2009). Phylogenetic affiliations of representative

170 sequences were analyzed via RDP Classifier against the SILVA (SSU115) 16S rRNA database

171 with a confidence threshold of 70\% (Quast et al., 2013). We used principal coordinates analysis

172 (PcoA) (Lozupone et al., 2005) to calculate beta diversity, and subsequently used ANOSIM to

173 confirm findings from the distance matrices.

174 To identify statistically significant taxonomic groups that differ between JS and AS, we

175 used Welch's t-tests (confidence interval method: Welch's inverted, $\mathrm{P}<0.05$ ) to compare

176 differences in species abundance between the two groups using the Software of the Statistical

177 Analysis of Metagenomic Profiles (STAMP) (Parks \& Beiko, 2010). We also used the linear

178 discriminant analysis (LDA) effect size (LEfSe) to identify significant associations between

179 bacterial taxa and host groups (JS and AS) (Segata et al., 2011). Metagenomic functional

180 composition was predicted from the latest Kyoto Encyclopedia of Genes and Genomes (KEGG) 
181 database (Kanehisa et al., 2012) using the Phylogenetic Investigation of Communities by

182 Reconstruction of Unobserved States (PICRUSt) approach (Langille et al., 2013).

183

184 RESULTS

185 Bacterial complexity of the snail gut flora

186 A total of 576,400 raw reads were generated using the Illumina Miseq sequence platform and

187251,072 high quality sequences were obtained (following quality control and sequence filtration).

188 The mean ( \pm standard deviation) number of sequences per sample was 31,384 \pm 4,292 (Table S1)

189 with an average length of $434 \pm 1.5$ basepairs. The 214,584 rarefied sequences were clustered

190 into 1,196 OTUs (mean number per sample: $890.75 \pm 43.80$ ), with 1,130 and 1,125 OTUs in JS

191 and AS, respectively. The representative sequences for all OTUs are available in Data S1. Ace,

192 Chao, Shannon, Simpson and Sobs indices indicate no significant differences in the diversity

193 between JS and AS populations ( $p>0.05$, student's T test) (Table 2). The plateau status of the

194 rarefaction curves indicated sufficient depth of sequencing (Fig. S2).

\section{Taxonomic composition of gut bacterial community}

196 We characterized the gut bacterial communities of snails. The OTUs that could not be assigned

197 to a specific genus are displayed using the highest taxonomic level that could be assigned (order, 198 class, or phyla).

199 Ten phyla accounted for $98.9 \%$ of the total sequences (Fig. 1A). Proteobacteria (JS: $36.0 \%$, 200 AS: 31.6\%) and Cyanobacteria (JS: 16.3\%, AS: 19.5\%) were the most dominant bacterial phyla, 201 followed by Chloroflexi (JS: 9.7\%, AS: 13.1\%), Firmicutes (JS: 14.4\%, AS: 6.7\%) and 202 Actinobacteria (JS: 8.1\%, AS: 12.6\%). Other phyla with lower abundance were Tenericutes (JS: 203 7.3\%, AS: 6.2\%), Bacteroidetes (JS: 3.4\%, AS: 2.0\%), Fusobacteria (JS: 1.3\%, AS: 1.2\%), and 
204 Verrucomicrobia (JS: 0.7\%, AS: 1.6\%). One phylum (JS: 1.9\%, AS: 4.6\%) was not classified.

205 Proteobacteria contained the largest number of OTUs (454), which belonged to the following

206 classes: alpha-, gamma-, beta-, delta-, and epsilon-proteobacteria (Fig. S3), followed by

207 Firmicutes (168), Cyanobacteria(149), Actinobacteria (122), Bacteroidetes (87), and Chloroflexi

208 (70). A particularly high abundance of Cyanobacteria was found in the gut of snails, which may

209 have originated from the snail's diet.

210 There were 53 identifiable bacterial families with $>1 \%$ abundance in at least one of the

211 samples (Fig. 1B). Among them, FamilyI_o_SubsectionIII (c_Cyanobacteria),

212 Rhodobacteraceae, Chloroflexaceae, Mycoplasmataceae, Chromatiaceae,

213 FamilyII_o_SubsectionII_ (c_Cyanobacteria), Cyanobacteria, Lachnospiraceae,

214 Ruminococcaceae, Caldilineaceae, Nocardioidaceae, Acetobacteraceae, Leptotrichiaceae, and

215 MNG7 were the most common families.

216 There were 54 genera with $>1 \%$ abundance in at least one of the samples and the sequences

217 of these genera constituted $55.6 \%$ of the total number of all sequences. Of all 54 genera, 36

218 genera were identifiable (Fig. 1C). The 15 most abundant classified genera were Paracoccus,

219 Pleurocapsa, Microcoleus, Thiodictyon, Leptolyngbya, Eubacterium, Subdoligranulum,

220 Nocardioides, Pseudomonas, Faecalibacterium, Chroococcidiopsism, Kluyvera, Rhodobacter,

221 Lemprocystis, and Gemmobacter, with abundances ranging from $1 \%$ to $9.9 \%$.

\section{Microbial community analysis}

223 Principal coordinate analysis (PcoA) was used to determine the similarities of gut microbial

224 communities between JS and AS. Unweighted UniFrac distance PcoA showed that juvenile snail

225 samples formed a distinct cluster and could be separated from adult snail samples (ANOSIM:

226 Unweighted unifrac, $\mathrm{p}$-value $=0.021, \mathrm{R}$-value $=0.677)$. In contrast, when we used weighted 
227 UniFrac to account for the abundance information, the samples did not apparently cluster into

228 two groups (ANOSIM: Weighted unifrac, $\mathrm{p}$-value $=0.199$, R-value $=0.198)($ Fig. 2).

229 We also assessed differences in species abundance between JS and AS populations. We

230 found no differences in the abundance of the vast majority of bacteria at both phyla and genera

231 levels (Fig. 3A and B). LEfSe analysis (threshold: 4.0) showed that two genera of bacteria were

232 significantly associated with JS, Ruminococcaceae (JS: 5.8\%; AS: 1.4\%), and Subdoligranulum

233 (JS: 3\%; AS: 0.6\%) (Fig. 3C)

\section{Bacterial community differences and similarities}

235 Venn analyses found that 1060 OTUs ( $88.7 \%$ of 1,196 OTUs identified) were shared between JS

236 and AS. In fact, 70 unique OTUs were found in JS and 65 were found in AS (Fig. S4). We found

237469 core OTUs in all snail samples representing $88.4 \%$ of all OTU sequences (Table S2).

238 Among these, 15 core OTUs had a mean abundance $>1 \%$, and supplied $33.9 \%$ of all OTU

239 sequences. The most abundant core bacterial genera were Mycoplasmataceae, Chloroflexaceae,

240 Paracoccus, Microcoleus, Pleurocapsa, Thiodictyon, Caldilineaceae, leptolyngbya, Eubacterium,

241 Subdoligranulum, and Nocardioides (Table S2).

\section{Functional predictions of bacterial communities}

243 The predicted genomic functions of $R$. auricularia bacterial community were performed using

244 PICRUSt. The level 1 KEGG pathways indicated a high abundance of predicted functions related

245 to metabolic pathways, environmental information processing, and genetic information

246 processing. The relative abundance of metabolic pathways accounted for 50.8\% (Fig. S5).

247 The level 2 KEGG pathway data (Fig. 4) indicated that the pathways related to membrane

248 transport, amino acid metabolism, carbohydrate metabolism, and xenobiotic biodegradation and

249 metabolism were enriched in both JS and AS samples, with average abundances of $12.4 \%$, 
$25010.5 \%, 10.0 \%$, and $5.7 \%$, respectively. Further examination of the carbohydrate metabolism

251 pathways indicated an abundance $(6.7 \%)$ of pathways related to both the starch and sucrose

252 metabolism, including functions for glycoside hydrolysis such as cellulose degradation (Fig. S6).

253 The KEGG pathways of energy metabolism and cell motility, and transcription showed

254 significant differences between JS and AS groups $(P<0.05)$. The pathways related to human

255 diseases (e.g. infectious and neurodegenerative diseases) were found to have low abundances.

256 Genetic pathways associated with xenobiotic biodegradation and metabolism maybe play a

257 role in environmental cleaning or bioremediation in the ecosystem. Results showed that the

258 intestinal microbiota were enriched with functions that were related to organic contaminant

259 metabolism (Figure S7). This included contaminants typically metabolized by the cytochrome

260 p450 family, including benzoate, toluene, aminobenzoate, naphthalene, polycyclic aromatic

261 hydrocarbons and other similar xenobiotics. Furthermore, some pathways that are typically

262 associated with the degradation of highly toxic matter were also present in high abundance,

263 including those associated with the degradation of dioxins, atrazine, xylene, bisphenol A, and

264 ethylbenzene. This indicated that the gut bacteria of snails may help to degrade anthropogenic

265 pollutants, which could otherwise be harmful to animals and humans.

\section{DISCUSSION}

$268 R$. auricularia is a freshwater herbivorous snail of great environmental and ecological 269 importance (AL-Sultan et al., 2017; Eckblad, 2010). In this study, snails were sampled during the 270 summer, at a time when the snails typically undergo rapid growth due to suitable temperatures 271 and abundant food supply (Guo et al., 2016; Zhang et al., 2018). To compare gut microbial 
272 communities at different growth stages, adult and juvenile snails were captured. To limit

273 differences of environmental conditions, all snails were sampled from the same aquatic area.

274 We characterized the gut bacterial community of the snail $R$. auricularia using next

275 generation sequencing technology. The alpha- diversity indices indicate a high diversity of the $R$.

276 auricularia bacterial community. The high proportion of shared OTUs and similarities between

277 the most abundant bacterial taxa indicates that adult and juvenile snails likely have similar gut

278 bacterial community structures. Using unweighted PCoA plot, samples were clustered according

279 to their growth stage (JS and AS clusters), indicating that the developmental stage may have an

280 effect on gut bacterial community. In contrast, as weighted PCoA showed, despite its high R

281 values, the clustering was not significant $(\mathrm{P}>0.05)$. This is at least in part due to abundance

282 information, which can obscure significant patterns of variation in the taxa that are present

283 (Lozupone et al., 2007; Chang et al., 2011), indicating that taking the abundance of bacterial

284 taxon into account revealed similarities between JS and AS populations that were not detected

285 solely by an examination of phylogenetic lineages. The obtained OTUs (1196) belonged to more

286 than 10 phyla (predominantly Proteobacteria followed by Cyanobacteria, Chloroflexi and

287 Firmicutes). At the phylum level, Proteobacteria was identified as the dominant bacteria in $R$.

288 auricularia bacterial community. Previously, Proteobacteria was also observed as most dominant

289 bacteria in other snails, such as Achatina fulica (Pawar et al., 2012), Helix pomatia (Nicolai et

290 al., 2015), Biomphalaria pfeifferi, Bulinus africanus, and Helisoma duryi (Van Horn et al., 2012).

291 However, at both the family and genus levels, there were differences between our study and the

292 results reported previously, i.e., the dominant bacteria for $H$. pomatia were pseudomonadaceae,

293 enterobacteriaceae and pantoea, for A. fulica, these were Citrobacter and Enterobacter. In

294 contrast, our results indicated Rhodobacteraceae, Chloroflexaceae, Mycoplasmataceae, 
295 Paracoccus, Thiodictyon, and Eubacterium as the most abundant gut bacteria. The differences of

296 bacterial communities between these snails may be caused by species, habitat, physiological

297 states, and environmental changes (Nicolai et al., 2015). The bacterial taxa present in our study

298 (e.g. Pseudomonas, Clostridiaceae, Lactococcus, Bacteroides, Flavobacteriaceae,

299 Mucilaginibacter, Citrobacter, Aeromonas, Acinetobacter, and Sulforospirillum) were also

300 previously reported in the gut of A. fulica (Cardoso et al., 2012b), which demonstrated the 301 occurrence of herbivore and plant-associated bacteria.

302 Cyanobacteria are widespread throughout aquatic areas and are the main source of energy

303 for snails (Qiao et al., 2018). Cyanobacteria were the second most dominant bacterial taxa in our

304 study: 147 OTUs were assigned to Cyanobacteria, which represented $12.3 \%$ of the total OTUs

305 and $17.9 \%$ of the total abundance. The second commonly detected OTU (OTU107) was

306 Chloroflexaceae, which is considered to be photosynthetic bacteria (Gupta, 2013). Similar to

307 other herbivores, the high abundance of Cyanobacteria was likely a result of incomplete 308 digestion of exogenous plants (Ye et al., 2014). Among this phylum, Family I (order Subsection

309 III) was most dominant among all measured snail samples (Fig. 1B), suggesting that Family I

310 (order Subsection III) may be an important dietary resource for $R$. auricularia. Many of the

311 Cyanobacteria bacteria found in the snail gut, including Leptolyngbya, Nostoc, and Pleurocapsa,

312 Microcoleus, Gemmobacter, Exiguobacterium, and Rubrobacter, are of environmental origin,

313 such as fresh water and soil (Hagemann et al., 2014; Lv et al., 2017; Strahsburger et al., 2018;

314 Albuquerque et al., 2014).

315 As the largest biomass on earth, cellulose and hemicellulose have the greatest potential for

316 the production of biofuels via hydrolytic processes (Lynd et al., 2008). Gut bacterial

317 communities play an important role in the digestion of cell walls and plant lignocelluloses 
318 because of the presence of glycoside hydrolases (Morrison et al., 2009). Many bacteria found in

319 our study, such as Paracoccus, Pseudomonas, Aeromonas, Stenotrophomonas, Citrobacter,

320 Bacillus, Micrococcus, Devosia, Shinella and Rhizobium, have previously been identified as

321 cellulolytic species, associated with carboxymethyl cellulase (CMCase) activity or avicelase

322 activity (Huang et al., 2012; Saha et al., 2006; Pawar et al., 2015). Paracoccus, Pseudomonas,

323 and Aeromonas were predominant bacteria in $R$. auricularia, indicating that they might be

324 important for the cellulose degradation process. Huang et al. (2012) reported that $70 \%$ of the

325 isolated cellulolytic bacteria from the gut of Holotrichia parallela larvae were Proteobacteria,

326 and some of the cellulolytic bacteria belonged to Actinobacteria, Firmicutes and Bacteroidetes,

327 which is similar to the findings of our study. The genera Klebsiella and Enterobacter were found

328 in the A. fulica gut at a dominant position among the cellulolytic bacterial community; however,

329 they were not found in our study (Pawar et al., 2015). Paracoccus, may not only be important

330 cellulolytic bacteria as described above, but also have been found to be a potential bacteria for

331 bioremediation of PAHs-contaminated soils (Teng et al., 2010). Although many members of

332 Pseudomonas are animal and plant pathogens, some members of the genus are able to degrade

333 chemical pollutants in the environment, such as polycyclic aromatic hydrocarbons, and carbon

334 tetrachloride (O'Mahony et al., 2006; Sepúlveda-Torres et al., 1999). As a member of the

335 Enterobacteriaceae family, Aeromonas inhabit fresh and brackish water and are responsible for

336 human intestinal diseases (Parker \& Shaw, 2011). However, in snails, Aeromonas are one of the

337 cellulolytic bacteria. In summary, in this study the cellulolytic bacteria found in $R$. auricularia

338 are not only centrally important due to their role in the degradation of cellulose and other plant

339 wall components, but they are also important due to their role in bioremediation of the ecosystem. 
341 Our results show that the most abundant OTU (OTU585) were affiliated with

342 Mycoplasmataceae, which belongs to the phylum Tenericute. Mycoplasma has been implicated

343 as an infectious species that can colonize humans and a wide range of animal species, causing

344 diseases in the hosts (Biondi et al., 2014). The predicted functions of infectious and human

345 diseases that were identified among the KEGG pathways could potentially be associated with the

346 genus Mycoplasma.

347 Although previous research has confirmed that bacterial communities vary during host

348 development and growth (from birth to adulthood) (Nistal et al., 2012; Stephens et al., 2016),

349 other studies have shown that the bacteria communities are relatively similar between juvenile

350 and adult stages in a variety of animal hosts (Xue et al., 2015; Hird et al., 2014). Our study also

351 showed that taking the abundance of bacterial taxon into account (weighted UniFrac) revealed

352 similarity of bacterial communities between JS and AS populations. The enrichment of

353 Faecalibacterium and Subdoligranulum (both belong to Ruminococcaceae) in JS and their poor

354 presence in AS is commonly found in many other animals ( $G U$ et al., 2013; Dethlefsena \&

355 Relman, 2011). These bacteria have been found to be highly beneficial to their hosts, by

356 producing butyrate and other short-chain fatty acids via fermentation of dietary fibers (Miquel et

357 al., 2013; Flint et al., 2012). These biomarkers may be important for JS in terms of improvement

358 of digestive ability, boosting their immune system, and other similar physiological functions ( $G U$

359 et al., 2013; Flint et al., 2012).

360 To understand the role of gut bacterial community in snails, we explored the function of gut

361 bacteria using PICRUSt (based on the 16rRNA gene data). The obtained results indicated that

362 the microbiome taxa are related to many physiological functions, which may aid their hosts

363 (Sommer \& Bäckhed, 2013). Previous studies with the snail A. fulica showed that many 
364 particular functional genes in the gut microbiota (e.g. genes associated with the production of

365 amino acids, fatty acids, cofactors, vitamins, and enzymes) are required by the hosts for plant

366 fiber degradation (Cardoso et al., 2012a). As recently reported by Joynson et al. (2017), 2,510

367 genes corresponding to glycoside hydrolase activity and 561 carbohydrate-binding modules were

368 identified in a total of 108,691 putative genes of the gut microbiome of the common black slug

369 Arion ater. The microbiotic function predicted in our study are also necessary for many

370 physiological functions. In fact, the richness of cellulolytic bacterial taxa could lead to the

371 isolation of bacterial cellulases from snails (Pinheiro et al., 2015). Furthermore, the discovery of

372 bacteria related to xenobiotic biodegradation illustrates the role of snails in the degradation of

373 environmental contaminants, indicating the potential application of the snail microbiota for

374 environmental cleaning, which was also found in other animal or environmental microbiota

375 (Yang et al., 2015; Zhou et al., 2016).

\section{CONCLUSIONS}

378 The use of advanced molecular technology offers a new method to study microbial communities

379 based on their DNA. In this study, we used the high-throughput sequencing technique to 380 investigate the bacterial diversity of individuals of the snail $R$. auricularia and predicted 381 metagenomic functions using PICRUSt. This work demonstrates that the phyla Proteobacteria,

382 Cyanobacteria, Chloroflexi,and Firmicutes were predominant in the microbial community. A

383 high number of OTU and genus diversity were shown. Growth and gonad development may

384 have influenced the taxonomic characteristics of the gut bacterial community without influencing

385 the predicted function of gut bacteria. For $R$. auricularia, the potential for isolating cellulolytic

386 bacteria and environmental cleaning are indicated by the abundant presence of cellulolytic 
387 bacteria and metagenomic functional predictions. Further research is required to better

388 characterize the interaction between gut flora and their hosts in snails such as $R$. auricularia.

389

390 REFERENCES

391 AL-Sultan EYA. 2017. Isolation, purification and identification of blue-green alga 392 Hapalosiphon aureus and evaluation of its histopathological effects on fresh water snail 393 Lymnaea auricularia. Journal of Applied Sciences 17: 61-71 DOI 10.3923/jas.2017.61.71.

Albuquerque L, Johnson MM, Schumann P, Rainey FA, Costa MSD. 2014. Description of two new thermophilic species of the genus Rubrobacter, Rubrobactercalidifluminis sp. nov. and Rubrobacter naiadicus sp. nov., and emended description of the genus Rubrobacter and the species Rubrobacter bracarensis. Systematic and Applied Microbiology 37:235-243 DOI 10.1016/j.syapm.2014.03.001.

Aronson HS, Zellmer AJ, Goffredi SK. 2016. The specific and exclusive microbiome of the deep-sea bone-eating snail, Rubyspira osteovora. Fems Microbiology Ecology 93:1-13 DOI 10.1093/femsec/fw250

Bargues MD, Vigo M, Horák P, Dvorák J, Patzner RA, Pointier JP, Jackiewicz M, MeierBrook C, Mas-Coma S. 2001. European Lymnaeidae (Mollusca: Gastropoda), intermediate hosts of trematodiases, based on nuclear ribosomal DNA ITS-2 seqeunces. Infection Genetics and Evolution 1:85-107 DOI 10.1016/S1567-1348(01)00019-3.

Biondi E, McCulloh R, Alverson B, Klein A, Dixon A. 2014. Treatment of mycoplasma pneumonia: a systematic review. Pediatrics 133:1081-1090 DOI 10.1542/peds.

Caporaso JG, Kuczynski J, Stombaugh J, Bittinger K, Bushman FD, Costello EK, Fierer N, Pena AG, Goodrich JK, Gordon JI, Huttley GA, Kelley ST, Knights D, Koenig JE, Ley 

7:335-336 DOI 10.1038/nmeth.f.303.

414 Cardoso AM, Cavalcante JJ, Cantão ME, Thompson CE, Flatschart RB, Glogauer A, 415 Scapin SM, Sade YB, Beltrão PJ, Gerber AL, Martins OB, Garcia ES, de Souza W, 416 Vasconcelos AT. 2012a. Metagenomic analysis of the microbiota from the crop of an 417 invasive snail reveals a rich reservoir of novel genes. PLOS ONE 7:e48505 DOI $418 \quad$ 10.1371/journal.pone.0048505.

419 Cardoso AM, Cavalcante JJ, Vieira RP, Lima JL, Grieco MA, Clementino MM, 420 Vasconcelos AT, Garcia ES, de Souza W, Albano RM, Martins OB. 2012b. Gut bacterial 421 communities in the giant land snail Achatina fulica and their modifcation by sugarcane-based 422

Carnevali O, Maradonna F, Gioacchini G. 2017. Integrated control of fish metabolism, wellbeing and reproduction: The role of probiotic. Aquaculture 472:144-155 DOI 10.1016/j.aquaculture.2016.03.037.

Chandler JA, James PM, Jospin G, Lang JM. 2014. The bacterial communities of Drosophila suzukii collected from undamaged cherries. PeerJ 2:e474 DOI 10.7717/peerj.474.

Chang Q, Luan Y, Sun F. 2011. Variance adjusted weighted UniFrac: a powerful beta diversity measure for comparing communities based on phylogeny. BMC Bioinformatics 12:118 DOI 10.1186/1471-2105-12-118.

431 Charrier MY, Gerard F, Gaillard-Martinie B, Kader A, Gerard A. 2006. Isolation and 432 characterization of cultivable fermentative bacteria from the intestine of two edible snails, Helix 
433 pomatia and Cornu aspersum (Gastropoda: Pulmonata). Biological Research 39:669-681 DOI 434 /S0716-97602006000500010.

435 Dennis KL, Wang Y, Blatner NR, Wang S, Saadalla A, Trudeau E, Roers A, Weaver CT, 436 Lee JJ, Gilbert JA, Chang EB, Khazaie K. 2013. Adenomatous polyps are driven by 437 microbe-instigated focal inflammation and are controlled by IL-10-producing T cells. Cancer $438 \quad$ Research 73:5905-5913 DOI 10.1158/0008-5472.

439 Dewitt TJ, Sih A, Hucko JA. 1999. Trait compensation and cospecialization in a freshwater 440 snail: size, shape and antipredator behaviour. Animal Behaviour 58:397-407 $441 \quad$ DOI 10.1006/anbe.1999.1158.

442 Dethlefsena L, Relman DA. 2011. Incomplete recovery and individualized responses of the 443 human distal gut microbiota to repeated antibiotic perturbation. (Supplement 1):4554-4561. DOI 10.1073/pnas.1000087107.

Dikkeboom R, Van der Knaap WP, Meuleman EA, Sminia T. 1985. A comparative study on the internal defense system of juvenile and adult Lymnaea stagnalis. Immunology 55:547553.

Eckblad J. 2010. Biomass and energy transfer by a specialized predator of aquatic snails. Freshwater Biology 6:19-21 DOI 10.1111/j.1365-2427.1976.tb01586.x.

451 Edgar RC, Haas BJ, Clemente JC, Christopher Q, Rob K, 2011. UCHIME improves 452 sensitivity and speed of chimera detection. Bioinformatics 27:2194-2200 DOI 453 10.1093/bioinformatics/btr381. 
454 Flint HJ, Scott KP, Louis P, Duncan SH. 2012. The role of the gut microbiota in nutrition and

455

456

457

458

459

460

461

462

463

464

465

466

467

468

469

470

471

472

473

474

475 health. Nature Reviews Gastroenterology \& Hepatology 9:577-589 DOI 10.1038/nrgastro.2012.156.

Gioacchini G, Lombardo F, Merrifield DL, Silvi S, Cresci A, Avella MA, Carnevali O. 2011. Effects of probiotics on zebra fish reproduction. Journal of Aquaculture \& Research Development S1:002 DOI 10.4172/2155-9546.S1-002.

Gu S, Chen D, Zhang JN, Lv X, Wang K, Duan LP, Nie Y, Wu XL. 2013. Bacterial community mapping of the mouse gastrointestinal tract. PLoS ONE 10:e74957 DOI 10.1371/journal.pone.0074957

Guo F, Kainz MJ, Valdez D, Sheldon F, Bunn SE. 2016. The effect of light and nutrients on algal food quality and their consequent effect on grazer growth in subtropical streams. Freshwater Science 35:1202-1212 DOI 10.1086/688092.

Gupta RS. 2013. Molecular markers for photosynthetic bacteria and insights into the origin and spread of photosynthesis. Advances in Botanical Research 66:37-66 DOI 10.1016/B978-012-397923-0.00002-3.

Hagemann M, Henneberg M, Felde VJ, Drahorad SL, Berkowicz SM, Henningsen PF, Kaplan A. 2015. Cyanobacterial diversity in biological soil crusts along a precipitation gradient, northwest Negev Desert, Israel. Microbial Ecology 70:219 DOI 10.1007/s00248014-0533-z

Hamelinck CN, Van Hooijdonk G, Faaij ACP. 2005. Ethanol from lignocellulosic biomass: techno-economic performance in short-, middle- and long-term. Biomass and Bioenergy 28:384-410. DOI 10.1016/j.biombioe.2004.09.002. 
476 Hird SM, Carstens BC, Cardiff SW, Dittmann DL, Brumfield RT. 2014. Sampling locality is

477 more detectable than taxonomy or ecology in the gut microbiota of the brood-parasitic

478 Brown-Headed Cowbird (Molothrus ater). PeerJ 2:e321 DOI 10.7717/peerj.321.

479 Jami E, Israel A, Kotser A, Mizrahi I. 2013. Exploring the bovine rumen bacterial community 480 from birth to adulthood. The ISME Journal 7:1069-1079 DOI 10.1038/ismej.2013.2.

481 Joynson R, Pritchard L, Osemwekha E. 2017. Metagenomic analysis of the gut microbiome of 482 the common black slug Arion ater in search of novel lignocellulose degrading enzymes. 483 Frontiers in microbiology 8:2181 DOI 10.3389/fmicb.2017.02181.

484 Kanehisa M, Goto S, Sato Y, Furumichi M, Tanabe M. 2012. KEGG for integration and 485 interpretation of large-scale molecular data sets. Nucleic Acids Research 40:109-114 DOI 486 10.1093/nar/gkr988.

Langille MGI, Zaneveld J, Caporaso JG, McDonald D, Knights D, Reyes, JA, Clemente JC, functional profiling of microbial communities using 16S rRNA marker gene sequences. Nature Biotechnology 31:814-821 DOI 10.1038/nbt.2676.

Llewellyn MS, McGinnity P, Dionne M, Letourneau J, Thonier F, Carvalho GR, Creer S, Derome N. 2015. The biogeography of the Atlantic salmon (Salmo salar) gut microbiome. The ISME Journal 10:1280-1284 DOI 10.1038/ismej.2015.189.

Lozupone CA, Knight R. 2005. UniFrac: a new phylogenetic method for comparing microbial 495 communities. Applied and Environmental Microbiology

71:8228-8235 DOI 
497 Lozupone CA, Hamady M, Kelley ST, Knight R. 2007. Quantitative and qualitative diversity

498 measures lead to different insights into factors that structure microbial communities. Applied 499 and Environmental Microbiology 73:1576-1585 DOI 10.1128/AEM.01996-06

500 Lv P, Luo J, Zhuang X, Zhang D, Huang Z, Bai Z. 2017. Diversity of culturable aerobic 501 denitrifying bacteria in the sediment, water and biofilms in Liangshui River of Beijing, China. $502 \quad$ Scientific reports 7:10032 DOI 10.1038/s41598-017-09556-9.

503 Lynd LR, Laser MS, Bransby D, Dale BE, Davison B, Hamilton R, Himmel M, Keller M, 504 McMillan JD, Sheehan J, Wyman CE. 2008. How biotech can transform biofuels. Nature 505 Biotechnology 26:169-172 DOI 10.1038/nbt0208-169.

Ma Y, Li B, Wang C, Shi Z, Sun Y, Sheng F, Zhang Y, Zhang W, Rao Y, Han S. 2014. 5HTTLPR polymorphism modulates neural mechanisms of negative self-reflection. Cerebral Cortex 24:2421-2429 DOI 10.1093/cercor/bht099.

Magoc T, Salzberg SL. 2011. FLASH: fast length adjustment of short reads to improve genome assemblies. Bioinformatics 27:2957-2963 DOI 10.1093/bioinformatics/btr507.

Miquel S, Martín, R, Rossi O, Humarán LGB, Chatel JM, Sokol H, Thomas M, Wells JM, Langella P. 2013. Faecalibacterium prausnitzii and human intestinal health. Current Opinion in Microbiology 16:255-261 DOI 10.1016/j.mib.2013.06.003.

514 Morrison M, Pope PB, Denman SE, McSweeney CS. 2009. Plant biomass degradation by gut microbiomes: more of the same or something new ? Current Opinion in Biotechnology 20:358-363 DOI 10.1016/j.copbio.2009.05.004.

517 Nayak SK. 2010. Role of gastrointestinal microbiota in fish. Aquaculture Research 41:1553518 1573 DOI 10.1111/j.1365-2109.2010.02546.x. 
519 Nicolai A, Rouland-Lefèvre C, Ansart A, Filser J, Lenz R, Pando A, Charrier M. 2015.

520 Inter-population differences and seasonal dynamic of the bacterial gut community in the 521 endangered land snail Helix pomatia (Gastropoda: Helicidae). Malacologia 59:177-190 DOI

$522 \quad 10.4002 / 040.059 .0101$.

523 Nistal E, Caminero A, Herrán AR, Arias L, Vivas S, Ruiz M, Jose C, Sara S, Miera L,

524 Arroyo P, Casqueiro J. 2012. Differences of small intestinal bacteria populations in adults 525 and children with/without celiac disease: Effect of age, gluten diet, and disease. 526 Inflammatory Bowel Diseases 18:649-656 DOI 10.1002/ibd.21830.

527 O'Mahony MM, Dobson AD, Barnes JD, Singleton I. 2006. The use of ozone in the 528 remediation of polycyclic aromatic hydrocarbon contaminated soil. Chemosphere 63:307314 DOI 10.1016/j.chemosphere.2005.07.018.

Pawar KD, Banskar S, Rane SD, Charan SS, Kulkarni GJ, Sawant SS, Ghate HV, Patole MS, Shouche YS. 2012. Bacterial diversity in different regions of gastrointestinal tract of Giant African Snail (Achatina fulica). Microbiology Open 1:415-426 DOI 10.1002/mbo3.38.

Pawar KD, Dar MA, Rajput BP, Kulkarni GJ. 2015. Enrichment and identification of cellulolytic bacteria from the gastrointestinal tract of Giant African snail, Achatina fulica. Applied Biochemistry \& Biotechnology 175:1971-1980 DOI 10.1007/s12010-014-1379-Z.

Parks DH, Beiko RG. 2010. Identifying biologically relevant differences between metagenomic communities. Bioinformatics 26: 715-721 DOI 10.1093/bioinformatics/btq041.

Parker JL, Shaw JG. 2011. Aeromonas spp. clinical microbiology and disease. Journal of Infection 62:109-118 DOI 10.1016/j.jinf.2010.12.003.

540 Pinheiro GL, Correa RF, Cunha RS, Cardoso AM, Chaia C, Clementino MM, Garcia ES,

541 de Souza W, Frasés S. 2015. Isolation of aerobic cultivable cellulolytic bacteria from 
different regions of the gastrointestinal tract of giant land snail Achatina fulica. Frontiers in Microbiology 6:860 DOI 10.3389/fmicb.2015.00860.

544 Qiao F, Lei K, Li Z, Wei Z, Liu Q, Yang L, He J, An L, Qi H, Cui S. 2018. Transcriptomic

545 responses of the freshwater snail (Parafossarulus striatulus) following dietary exposure to

546 cyanobacteria. Science of the Total Environment 624:153-161 DOI

$547 \quad$ 10.1016/j.scitotenv.2017.12.112.

548 Quast C, Pruesse E, Yilmaz P, Gerken J, Schweer T, Yarza P, Peplies J, Glockner FO. 2013.

549 The SILVA ribosomal RNA gene database project: improved data processing and web-based

$550 \quad$ tools. Nucleic Acids Research 41:D590-D596 DOI 10.1093/nar/gks 1219.

551 Saha S, Roy RN, Sen SK, Ray AK. 2006. Characterization of cellulase-producing bacteria from

552 the digestive tract of tilapia, Oreochromis mossambica (Peters) and grass carp,

553 Ctenopharyngodon idella (Valenciennes). Aquaculture Research 37:380-388 DOI

$554 \quad$ 10.1111/j.1365-2109.2006.01442.x.

555 Schloss PD, Westcott SL, Ryabin T, Hall JR, Hartmann M, Hollister EB, Lesniewski RA,

556 Oakley BB, Parks DH, Robinson CJ, Sahl JW, Stres B, Thallinger GG, Van Horn DJ,

557 Weber CF. 2009. Introducing mothur: open-source, platform-independent, community-

558 supported software for describing and comparing microbial communities. Applied and

559 Environmental Microbiology 75:7537-7541 DOI 10.1128/AEM.01541-09.

560 Schamp B, Horsák M, Hájek M. 2010. Deterministic assembly of land snail communities

561 according to species size and diet. Journal of Animal Ecology 79:803-810

562 DOI 10.1111/j.1365-2656.2010.01685.x. 
563 Segata N, Izard J, Waldron L, Gevers D, Miropolsky L, Garrett WS, Huttenhower C. 2011.

564 Metagenomic biomarker discovery and explanation. Genome Biology 12: R60 DOI $565 \quad 10.1186 / \mathrm{gb}-2011-12-6-r 60$.

566 Sepúlveda-Torres LC, Rajendran N, Dybas MJ, Criddl CS. 1999. Generation and initial 567 characterization of Pseudomonas stutzeri KC mutants with impaired ability to degrade carbon tetrachloride. Archives of Microbiology 171:424-429 DOI 10.1007/s002030050729.

Soldánová M, Selbach C, Sures B, Kostadinova A, Pérez-del-Olmo A. 2010. Research larval trematode communities in Radix auricularia and Lymnaea stagnalis in a reservoir system of the Ruhr River. Parasites Vectors 3:56 DOI 10.1186/1756-3305-3-56.

Sommer F, Bäckhed F. 2013. The gut microbiota-masters of host development and physiology. Nature Reviews Microbiology 11:227-238 DOI 10.1038/nrmicro2974.

Stephens WZ, Burns AR, Stagaman K, Wong S, Rawls JF, Guillemin K, Bohannan BJM. 2016. The composition of the zebrafish intestinal microbial community varies across development. The ISME Journal 10:644-654 DOI 10.1038/ismej.2015.140.

Stift M, Michel E, Sitnikova TY, Mamonova EY, Sherbakov DY. 2004. Palaearctic gastropod gains a foothold in the dominion of endemics: range expansion and morphological change of Lymnaea (Radix) auricularia in Lake Baikal. Hydrobiologia 513:101-108 DOI 10.1023/B:hydr.0000018175.37771.d6.

581 Strahsburger E, Zapata F, Pedroso I, Fuentes D, Tapia P, Ponce R, Valdes J. 2018. Draft 582 genome sequence of Exiguobacterium aurantiacum strain PN47 isolate from saline ponds, 583 known as "Salar del Huasco", located in the Altiplano in the North of Chile. Brazilian 584 Journal of Microbiology 49:7-9 DOI 10.1016/j.bjm.2017.03.011 
585 Vesbach CT, King K, Horn DV, Larkin K, Neiman M. 2016. Distinct bacterial microbiomes

586 in sexual and asexual Potamopyrgus antipodarum, a New Zealand freshwater snail. PLoS ONE 11:e0161050 DOI 10.1371/journal.pone.0161050.

588 Teng Y, Luo YM, Sun MM, Liu ZJ, Li ZG, Christie P. 2010. Effect of bioaugmentation by 589 Paracoccus sp. strain HPD-2 on the soil microbial community and removal of polycyclic aromatic hydrocarbons from an aged contaminated soil. Bioresource Technology 101:34373443 DOI 10.1016/j.biortech.2009.12.088.

Vasileva SY. 2012. Shell size of the freshwater snail Radix auricularia (Linnaeus, 1758) collected from water vegetation: a case study from South-East Bulgaria. Ecologia Balkanica 1:111-115.

Van Horn DJ, Garcia JR, Loker ES, Mitchell KR, Mkoji GM, Adema CM, VESBACH CDT. 2012. Complex intestinal bacterial communities in three species of Planorbid snails. Journal of Molluscan Study 78:74-80 DOI 10.1093/mollus/eyr038.

Xue Z, Zhang W, Wang L, Hou R, Zhang M, Fei L, Zhang X, Huang H, Bridgewater LC, Jiang Y, Jiang C, Zhao L, Pang X, Zhang Z. 2015. The bamboo-eating giant panda harbors a carnivore-like gut microbiota, with excessive seasonal variations. mBio 6:e0002215 DOI 10.1128/mBio.00022-15.

602 Yang Y, Yang J, Wu WM, Zhao J, Song Y, Gao L, Yang R, Jiang L. 2015. Biodegradation 603 and mineralization of polystyrene by plastic-eating mealworms: Part 2. Role of gut 604 microorganisms. Environmental Science Technology 49: 12087-12093 DOI 605 10.1021/acs.est.5b02663. 
606 Ye L, Amberg J, Chapman D, Gaikowski M, Liu WT. 2014. Fish gut microbiota analysis

607 differentiates physiology and behavior of invasive Asian carp and indigenous American fish.

608 The ISME Journal 8:541-551 DOI 10.1038/ismej.2013.181.

609 Zhang P, Blonk BA, van den Berg RF, and Bakker ES. 2018. The effect of temperature on

610 herbivory by the omnivorous ectotherm snail Lymnaea stagnalis. Hydrobiologia 812:147-

$611 \quad 155$ DOI 10.1007/s10750-016-2891-7.

612 Zhou C, Ontiveros-Valencia A, Wang ZC, Maldonado J, Zhao HP, Brown RK, Rittmann

613 BE. 2016. Palladium recovery in a H2-based membrane biofilm reactor: formation of $\operatorname{Pd}(0)$

614 nanoparticles through enzymatic and autocatalytic reductions. Environmental Science

615 Technology 50:2546-2555 DOI 10.1021/acs.est.5b05318. 


\section{Figure 1}

\section{Relative abundance of bacterial communities in $R$. auricularia samples.}

(A) Phylum level, all remaining taxa with abundance $<1 \%$ are summarized as other. (B) Family level (or the nearest identifiable phylogenetic level), all remaining taxa with abundance $<5 \%$ are summarized as other.

(C) Genus level (or the nearest identifiable phylogenetic level), all remaining taxa with abundance $<5 \%$ are summarized as other.

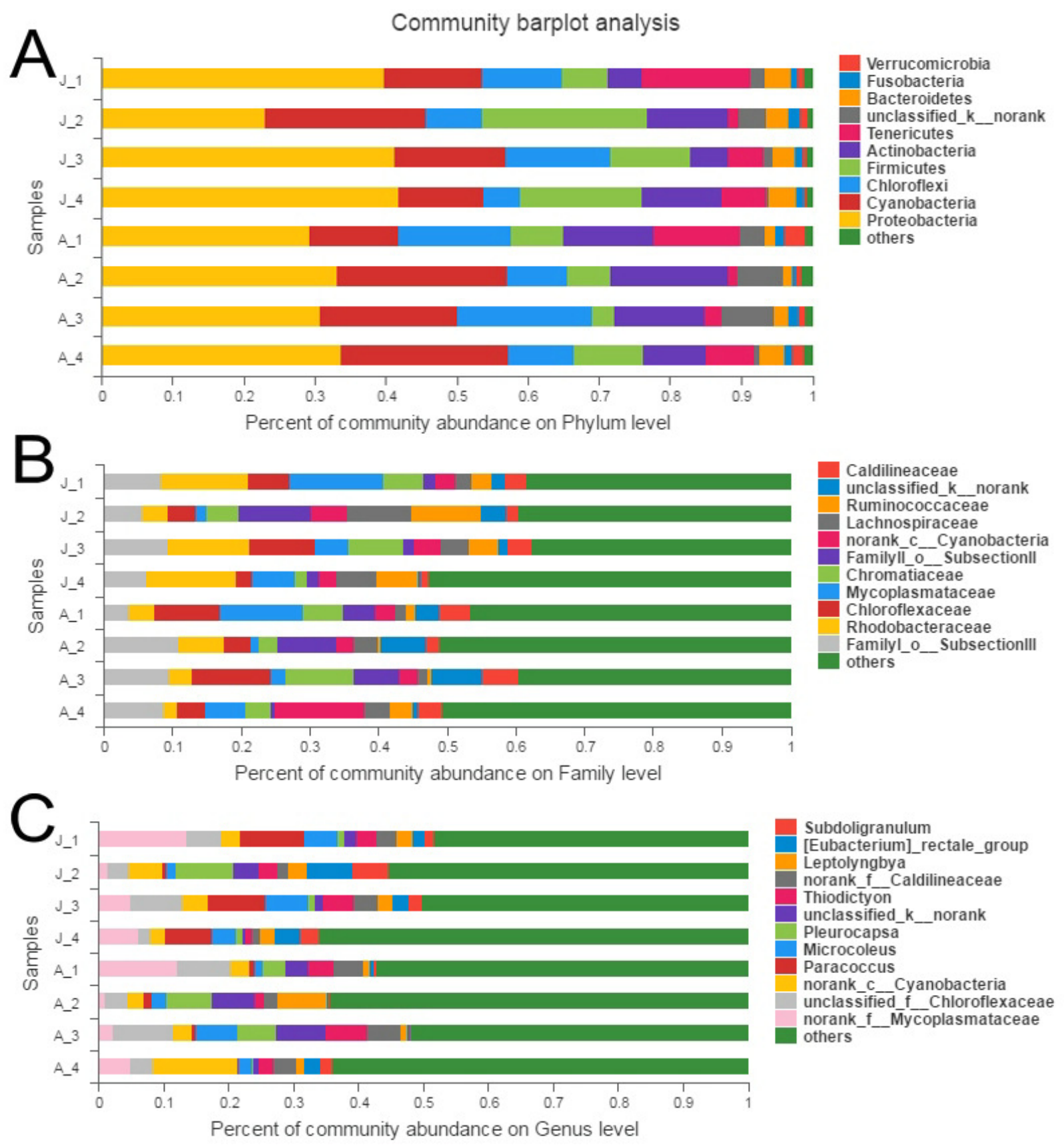


Figure 2

Unweighted uniFrac principal coordinate analysis of the snail bacterial communities.

The juvenile snails are shown by $\mathrm{J}, \mathrm{J} 2, \mathrm{J3}, \mathrm{J4}$, adult snails are shown by $\mathrm{A1}, \mathrm{A2}, \mathrm{A3}, \mathrm{A4}$.

ANOSIM: $p$-value $=0.021$, R-value $=0.677$.

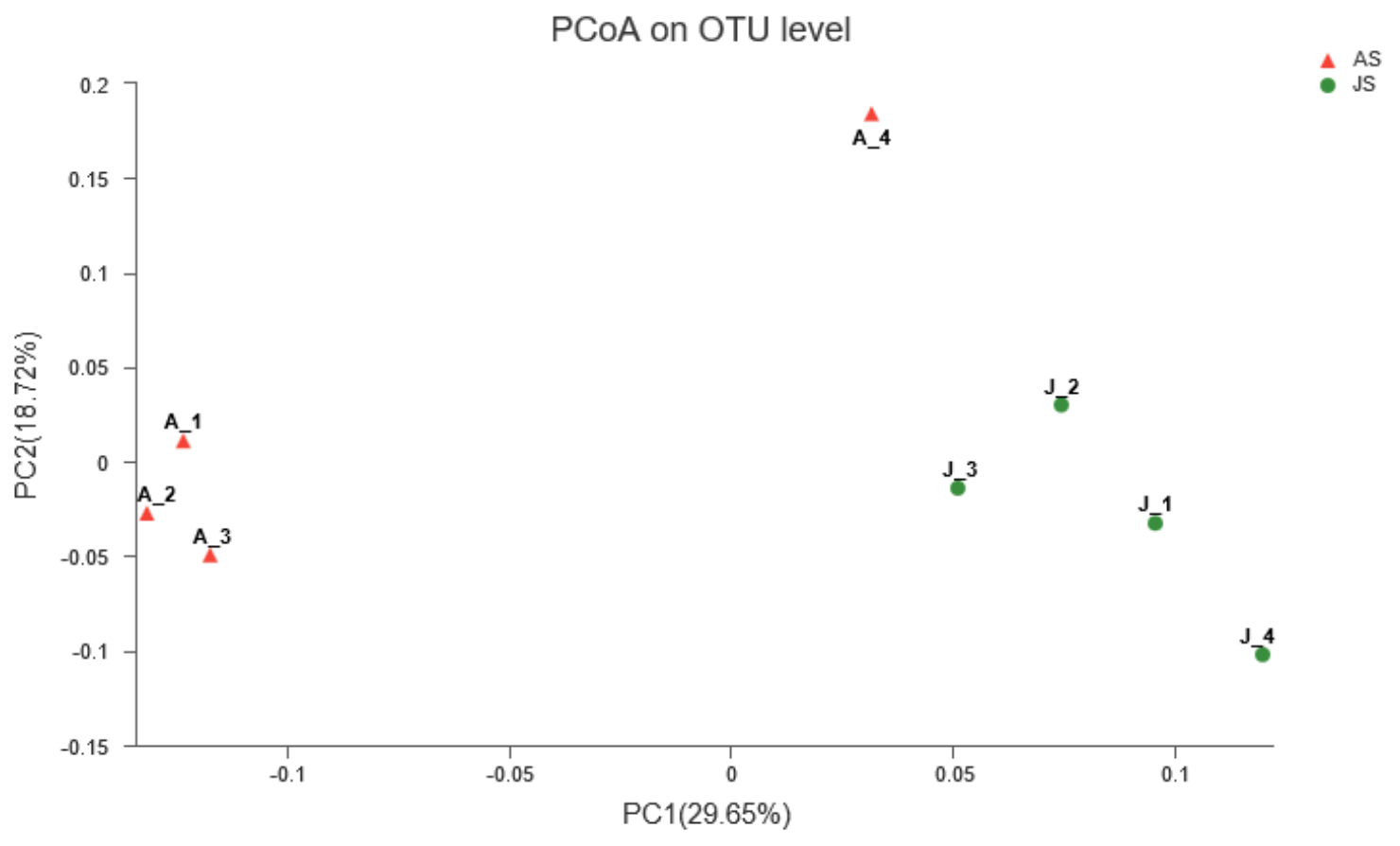




\section{Figure 3}

Taxonomic difference between juvenile and adult groups.

(A) Wilcoxon rank-sum test bar plot of bacterial phyla. (B) Wilcoxon rank-sum test bar plot of bacterial core genera. (C) Diagram of significant associations between gut bacterial taxa and snail population (linear discrimination algorithm LEFSe, Threshold $=4.0$ ). AS represented adult snails and JS represented juvenile snails. 

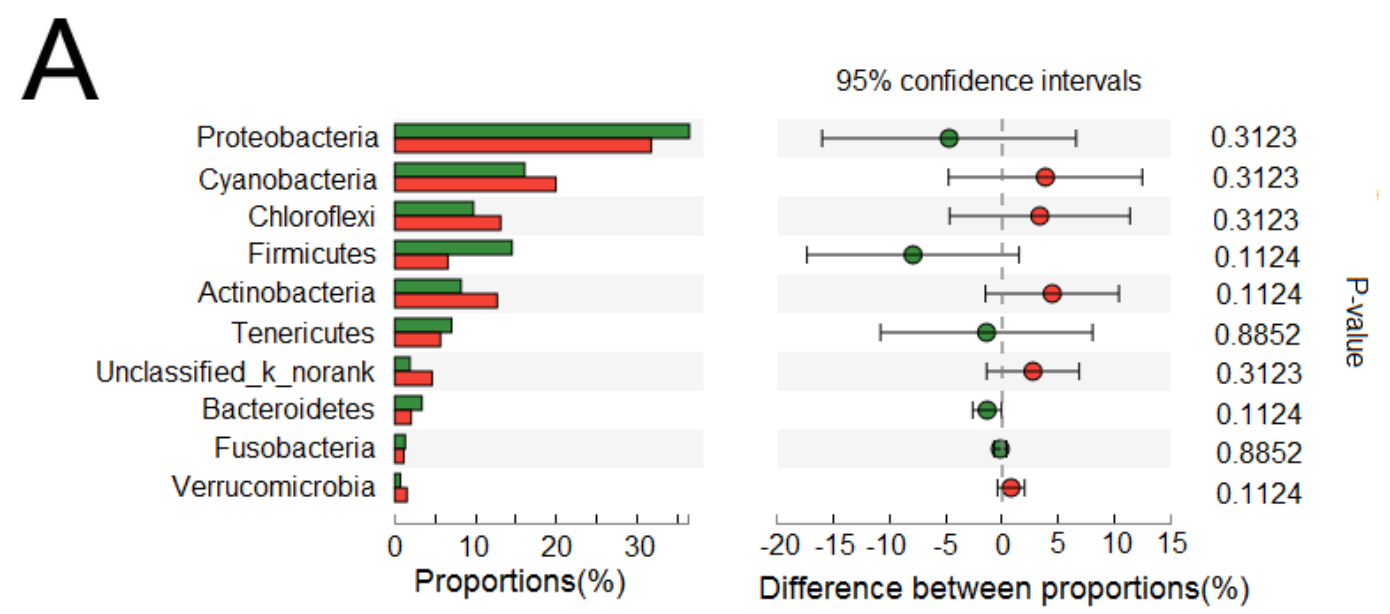

- JS

AS

B

norank_f_Mycoplasmataceae unclassified_f_Chloroflexaceae norank_c_Cyanobacteria Paracoccus Microcoleus Pleurocapsa unclassified_k_norank Thiodictyon norank_f_Caldilineaceae Leptolyngbya [Eubacterium]_rectale_group E Subdoligranulum Nocardioides norank_o_JG30-KF-CM45 norank_f_MNG7 unclassified_f_Leptotrichiaceae Pseudomonas norank_o_PeM15 Faecalibāterium unclassified_f_Rhodobacteraceae

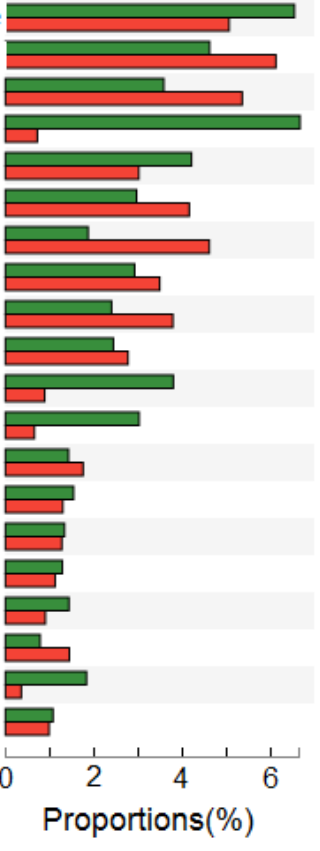

$95 \%$ confidence intervals

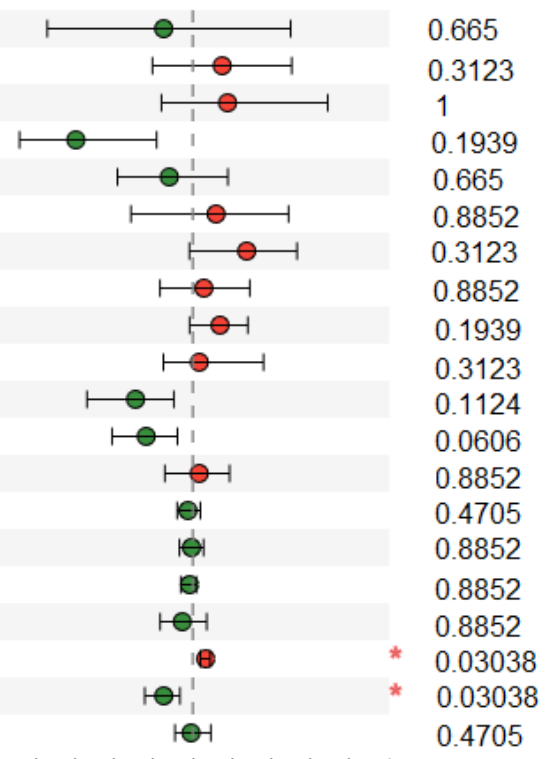

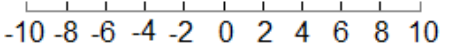

Difference between proportions(\%)

$\frac{\prod}{\grave{D}}$

LEfSe Bar

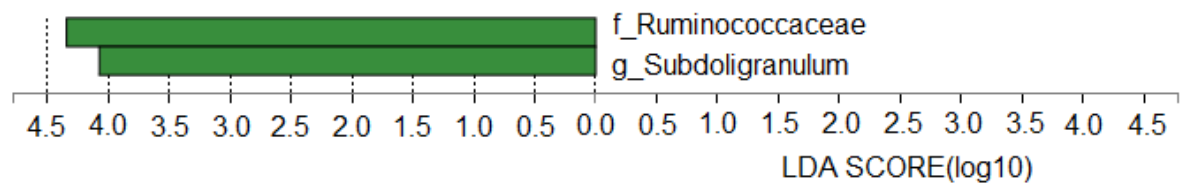




\section{Figure 4}

\section{PICRUSt predictions of the functional composition of snails microbiome.}

\section{The left list represents KEGG pathway at level 1, middle list represents KEGG pathway at}

level 2, the right list represents the abundance of each function pathway. JS: juvenile snails,

\section{AS: adult snails.}

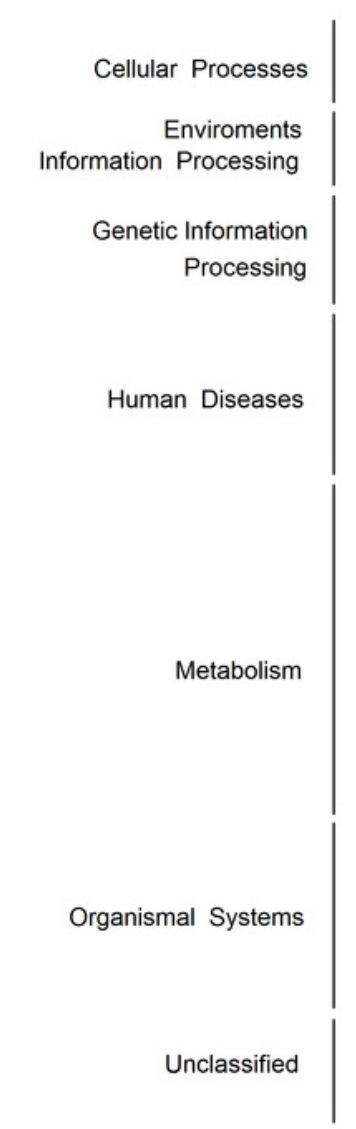

KEGG_Pathways Level 1

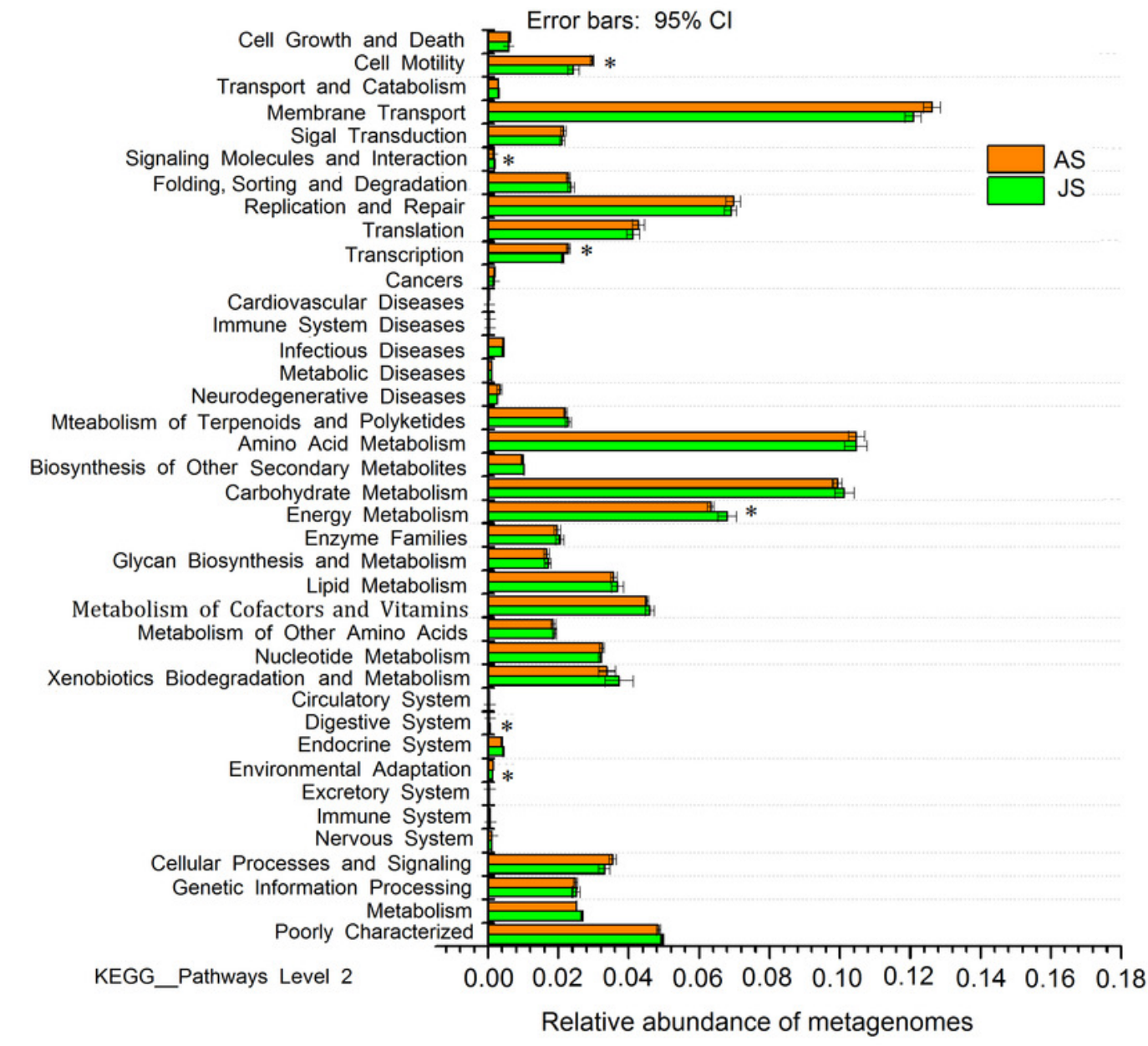


Table $\mathbf{1}$ (on next page)

DNA yield and quality of the snail bacteria 


\begin{tabular}{llll}
\hline samples & concentration $(\mathrm{ng} / \mu \mathrm{l})$ & OD260/280 & OD260/230 \\
\hline AS_1 & 78.10 & 1.96 & 0.36 \\
AS_2 & 81.20 & 1.99 & 0.14 \\
AS_3 & 69.40 & 2.08 & 0.10 \\
AS_4 & 320.10 & 1.92 & 0.68 \\
JS_1 & 92.90 & 1.96 & 0.43 \\
JS_2 & 300.50 & 1.97 & 0.44 \\
JS_3 & 81.80 & 1.93 & 0.77 \\
JS_4 & 207.90 & 1.96 & 0.35 \\
\hline
\end{tabular}


Table 2 (on next page)

Alpha-diversity of the bacterial communities in $R$. auricularia 
1

2

\begin{tabular}{cccccc}
\hline & Ace & Chao & Shannon & Simpson & Sobs \\
\hline Juvenile snails & $997.78 \pm 36.38$ & $1009.20 \pm 44.63$ & $5.00 \pm 0.22$ & $0.024 \pm 0.009$ & $901.75 \pm 25.9$ \\
Adult snails & $972.78 \pm 39.27$ & $974.77 \pm 48.68$ & $5.12 \pm 0.22$ & $0.019 \pm 0.007$ & $880 \pm 59.364$ \\
P-value & 0.67 & 0.47 & 0.47 & 0.48 & 0.67 \\
\hline
\end{tabular}

